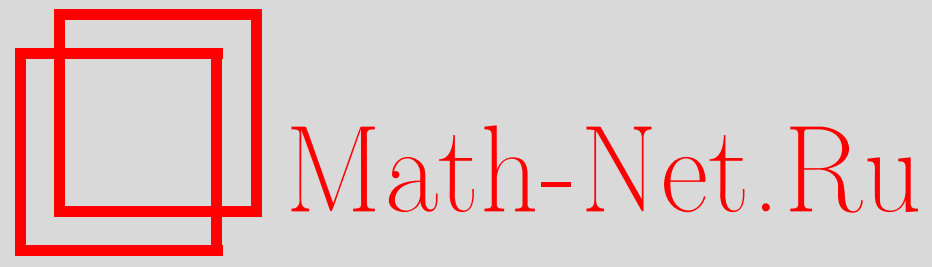

В. И. Арнольд, Комплексный лагранжев грассманиан, Функи. анализ и его прил., 2000, том 34, выпуск $3,63-65$

DOI: https://doi.org/10.4213/faa312

Использование Общероссийского математического портала MathNet.Ru подразумевает, что вы прочитали и согласны с пользовательским соглашением

http://www. mathnet.ru/rus/agreement

Параметры загрузки:

IP : 54.224 .135 .184

26 апреля 2023 г., 11:56:56

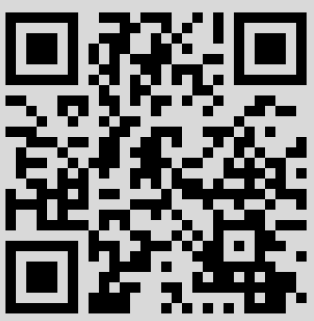




\title{
Комплексный лагранжев грассманиан
}

\author{
(c) 2000. В. И. Арнольд
}

Многообразие лагранжевых подпространств векторного симплектического пространства $\mathbb{R}^{2 n}$ есть $U(n) / O(n)$ (см. [1]). Естественно возникает вопрос о комплексном и кватернионном вариантах этих понятий (см. [2]). В настоящей работе показано, что комплексным аналогом является многообразие унитарных матриц $U(n)$. Это многообразие оказывается компактификацией пространства эрмитовых матриц в таком же смысле, в каком вещественное лагранжево многообразие Грассмана компактифицирует пространство симметрических матриц.

1. Комплексификация теоремы Дарбу. В комплексном векторном пространстве $\mathbb{C}^{n}$ вместо $\mathbb{C}$-симплектической структуры рассмотрим следующий ее аналог.

ОПРеДЕЛЕнИЕ. Симплектоидной структурой в $\mathbb{C}^{n}$ называется вещественная симплектическая структура $\Omega$, инвариантная относительно умножения на комплексные числа, по модулю равные $1: \Omega(\lambda \xi, \lambda \eta)=\Omega(\xi, \eta)$ при $|\lambda|=1$.

Теорема. Такая структура $\Omega$ существует и задается формулой $\Omega=$ $\sum \pm x_{k} \wedge y_{k}$ в подходящей системе координат $z_{k}=x_{k}+$ iу $y_{k}$ комплексного векторного пространства $\mathbb{C}^{n}$.

ДокАЗАТЕльство. Умножения на $e^{i t}$ образуют однопараметрическую группу линейных симплектических преобразований в $\mathbb{R}^{2 n}$, т. е. гамильтонов поток с квадратичным гамильтонианом $H$. Вещественным линейным симплектическим преобразованием такой гамильтониан приводится к форме

$$
H=\sum\left( \pm\left(p_{k}^{2}+q_{k}^{2}\right) / 2\right), \quad \Omega=\sum p_{k} \wedge q_{k},
$$

так как собственные частоты равны \pm 1 вследствие $2 \pi$-периодичности колебаний.

Инвариантные координатные плоскости $\left(p_{k}, q_{k}\right)$ - комплексные прямые (так как колебания состоят в умножении на $\left.e^{i t}\right)$. Симплектические ориентации $p_{k} \wedge q_{k}$ этих плоскостей могут совпадать либо не совпадать с комплексными ориентациями (заданными реперами вида $\xi, i \xi)$. Если комплексная координата на плоскости $\left(p_{k}, q_{k}\right)$ есть $z_{k}=x_{k}+i y_{k}$, то $x_{k} \wedge y_{k}=c_{k} p_{k} \wedge q_{k}$. Для $z_{k}(t)=e^{i t} \xi_{k}$ имеем $\dot{z}_{k}=i z_{k}, \dot{x}_{k}=-y_{k}, \dot{y}_{k}=x_{k}$, а для $H= \pm\left(p_{k}^{2}+q_{k}^{2}\right) / 2$ имеем $\dot{p}_{k}=\mp q_{k}$, $\dot{q}_{k}= \pm p_{k}$; поэтому $p_{k} \pm i q_{k}=c_{k}\left(x_{k}+i y_{k}\right)=c_{k} z_{k}$. Линейной заменой $Z_{k}=c_{k} z_{k}$ добиваемся, чтобы $p_{k} \pm i q_{k}=Z_{k}=X_{k}+i Y_{k}$, так что $d p_{k} \wedge d q_{k}= \pm d X_{k} \wedge d Y_{k}$, т. е. мы привели $\Omega$ к искомой канонической форме.

2. Приспособленная $\mathbb{C}$-симплектическая структура. Рассмотрим билинейную форму $S(\xi, \eta)=\Omega(i \xi, \eta)$, где $\Omega$ есть $\mathbb{C}$-симплектоидная структура. Форма $S$ симметрическая, так как $S(\eta, \xi)=\Omega(i \eta, \xi)=\Omega(-\eta, i \xi)=-\Omega(\eta, i \xi)=$ $\Omega(i \xi, \eta)=S(\xi, \eta)$. Сигнатура соответствующей квадратичной формы $S(\xi, \xi)$ является (единственным) инвариантом $\mathbb{C}$-симплектоидной структуры (она определяется числом минусов в нормальной форме в теореме). 
ОПРЕДЕЛЕНИЕ. Приспособленной $\mathbb{C}$-симплектической структурой в $\mathbb{C}^{2 n}$ называется симплектоидная структура, для которой сигнатура квадратичной формы $S$ равна нулю (т.е. в нормальной форме число минусов равно числу плюсов).

По теореме такая структура единственна (с точностью до комплексного линейного преобразования).

Пример. Обозначим слагаемые в разложении вектора $\xi \in \mathbb{C}^{2 n}=\mathbb{C}_{1}^{n} \oplus \mathbb{C}_{2}^{n}$ через $x \in \mathbb{C}_{1}^{n}, y \in \mathbb{C}_{2}^{n}$ и аналогичные слагаемые для вектора $\eta$ через $v \in \mathbb{C}_{1}^{n}$, $w \in \mathbb{C}_{2}^{n}$.

Будем обозначать знаком $\langle$,$\rangle эрмитово скалярное произведение векторов$ из $\mathbb{C}_{1}^{n}$ на векторы из $\mathbb{C}_{2}^{n}$ (например, $\langle x, w\rangle=\sum x_{k} \overline{w_{k}}$ ).

Рассмотрим в $\mathbb{C}^{2 n}$ эрмитову форму $\omega(\xi, \eta)=\langle x, w\rangle+\langle y, v\rangle \in \mathbb{C}$. Очевидно, $\omega(\eta, \xi)=\bar{\omega}(\xi, \eta), \omega(\lambda \xi, \eta)=\lambda \omega(\xi, \eta)$; поэтому $\omega(\lambda \xi, \lambda \eta)=\omega(\xi, \eta)$ при $|\lambda|=1$, так что форма $\omega S^{1}$-инвариантна.

Обозначим вещественную и мнимую части формы $\omega$ через $S$ и $\Omega$, так что $S(\xi, \eta) \in \mathbb{R}, \Omega(\xi, \eta) \in \mathbb{R}$,

$$
\omega(\xi, \eta)=S(\xi, \eta)+i \Omega(\xi, \eta)
$$

ПРЕДЛОЖЕНИЕ. Структура $\Omega$ является приспособленной $\mathbb{C}$-симплектической структурой в $\mathbb{C}^{2 n}$.

Вследствие уже доказанной единственности наша формула доставляет нормальную форму любой приспособленной $\mathbb{C}$-симплектической структуры.

По определению формы $\omega$

$$
\begin{aligned}
S(\xi, \eta) & =\operatorname{Re} x \operatorname{Re} w+\operatorname{Im} x \operatorname{Im} w+\operatorname{Re} y \operatorname{Re} v+\operatorname{Im} y \operatorname{Im} v=S(\eta, \xi), \\
\Omega(\xi, \eta) & =\operatorname{Im} x \operatorname{Re} w-\operatorname{Re} x \operatorname{Im} w+\operatorname{Im} y \operatorname{Re} v-\operatorname{Re} y \operatorname{Im} v=-\Omega(\eta, \xi), \\
\Omega(i \xi, \eta) & =\operatorname{Re} x \operatorname{Re} w+\operatorname{Im} x \operatorname{Im} w+\operatorname{Re} y \operatorname{Re} v+\operatorname{Im} y \operatorname{Im} v=S(\xi, \eta)
\end{aligned}
$$

поэтому

$$
S(i \xi, \eta)=\Omega(-\xi, \eta)=-\Omega(\xi, \eta) .
$$

Ясно, что $\Omega$ - кососимметрическая невырожденная форма, а $S$ - симметрическая форма сигнатуры 0 (тоже невырожденная): равенство сигнатуры нулю видно, например из формулы

$$
4 S(\xi, \eta)=\|x+w\|^{2}-\|x-w\|^{2}+\|y+v\|^{2}-\|y-v\|^{2} .
$$

Предложение доказано.

Введем обозначения для $n$-компонентных векторов

$$
x-y=X, \quad x+y=Y, \quad v-w=V, \quad v+w=W .
$$

Мы построили новое разложение $\mathbb{C}^{2 n}=C_{3}^{n} \oplus \mathbb{C}_{4}^{n}\left(X \in \mathbb{C}_{3}^{n}, Y \in \mathbb{C}_{4}^{n}, V \in \mathbb{C}_{3}^{n}\right.$, $W \in \mathbb{C}_{4}^{n}$ - новые «компоненты» векторов $\xi$ и $\left.\eta\right)$. Заметим, что $2 x=X+Y$, $2 y=-X+Y, 2 v=V+W, 2 w=-V+W$. Следовательно, форма $\omega$ имеет вид

$$
4 \omega(\xi, \eta)=4\langle x, w\rangle+4\langle y, v\rangle=2\langle Y, W\rangle-2\langle X, V\rangle .
$$

3. Лагранжевы плоскости. Подпространство $L \mathbb{C}$-размерности $n$ в $\mathbb{C}^{2 n}$ называется $\Omega$-лагранжевым, если $\Omega(\xi, \eta)=0$ для любых векторов $\xi, \eta$ из $L$. Оно называется $\omega$-лагранжевым, если $\omega(\xi, \eta)=0$ для любых векторов $\xi, \eta$ из $L$. 
ПРЕДЛОЖЕНИЕ. Подпространство $\Omega$-лагранжево, если и только если оно $\omega$-лагранжево.

ДокАЗАтельство. Мы уже видели выше, что $\omega(\xi, \eta)=\Omega(i \xi, \eta)+i \Omega(\xi, \eta)$. Поскольку подпространство комплексное, $i \xi$ принадлежит ему вместе с $\xi$; поэтому если $\Omega=0$, то и $\omega=0$. Если же $\omega=0$, то и ее мнимая часть $\Omega$ тоже есть 0 (как и ее вещественная часть, $S$ ).

ПрЕДЛОЖЕНИЕ. Пересечение любого лагранжева подпространства $L$ c плоскостью $x=y($ m. е. $X=0)$ нулевое.

ДокАЗАтЕЛЬСТво. Имеем $\omega(\xi, \xi)=2\langle x, x\rangle=0$ для любого $\xi$ из $L \cap(x=y)$; поэтому $x=0$.

СлЕДСтвИЕ. Любое лагранжево подпространство $L$ в $\mathbb{C}^{2 n}$ является графиком некоторого комплексно-линейного оператора $U: \mathbb{C}_{3}^{n} \rightarrow \mathbb{C}_{4}^{n}$, m. е. задается уравнением $Y=U X$.

Действительно, по предыдущему предложению $L$ является сечением расслоения $\mathbb{C}^{2 n} \rightarrow \mathbb{C}_{3}^{n}$ со слоем $\mathbb{C}_{4}^{n}$.

ТЕоремА. График оператора $U$ является лагранжевьлм тогда и только тогда, когда оператор $U$ является унитарньлм.

ДокАЗАТЕЛЬСтво. По формуле $(*)$

$$
4 \omega(\xi, \eta)=2\langle Y, W\rangle-2\langle X, V\rangle .
$$

Если $Y=U X, W=U V$, то условие лагранжевости $(\omega=0)$ принимает вид

$$
\langle U X, U V\rangle=\langle X, V\rangle
$$

для любых $X, V$ из $\mathbb{C}_{3}^{n}$, что и означает унитарность оператора $U$.

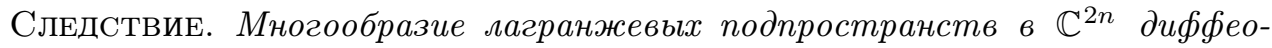
морфно группе унитарных матрии $U(n)$.

Диффеоморфизм сопоставляет матрице $U$ график оператора $U$.

ЗАмЕЧАНИЕ. Если комплексная плоскость является графиком оператора $y=$ $A x$, то условие лагранжевости $\omega(\xi, \eta)=\langle x, w\rangle+\langle y, v\rangle=0$ принимает вид $\langle x, A v\rangle+\langle A x, v\rangle=0$, т. е. $A^{*}=-A$ (оператор косоэрмитов). Связь между операторами $A$ и $U$ дается преобразованием Кэли (уже встречавшимся по этому поводу в [1]):

$$
A=(U-1) /(U+1), \quad U=(1+A) /(1-A) .
$$

Таким образом, унитарная группа оказывается компактификацией векторного (вещественного) пространства косоэрмитовых матриц (соответствующих унитарным матрицам без собственного числа -1$)$.

\section{ЛИТЕРАТУРА}

1. Арнольд В. И. Функц. анализ и его прил., 1, вып. 1, 1-14 (1967). 2. Arnold V. I. In: Mathematics: Frontiers and Perspectives (Arnold V., Atiyah M., Lax P., Mazur B., eds.), IUM, Amer. Math. Soc., 2000, pp. 403-416. 